\title{
Outils numériques soutenant les processus de régulation dans une communauté de coélaboration de connaissances
}

\author{
Digital tools sustaining regulation processes in a knowledge \\ building community
}

\section{Herramientas numéricas para apoyar los procesos de regulación en una comunidad de codesarrollo de conocimiento}

Michelle Deschênes, doctorante

Université Laval, Canada

michelle.deschenes@fse.ulaval.ca

Mélanie Tremblay, doctorante

Université Laval, Canada

melanie.tremblay.50@ulaval.ca

RÉSUMÉ

Dans le cadre d'un cours universitaire aux cycles supérieurs, six étudiants en sciences de l'éducation ont coélaboré sur le thème de l'équité numérique en présence et en ligne à l'aide du Knowledge Forum et de Google Drive. À la suite de cette expérience, une question s'est posée : comment maximiser le fonctionnement d'une communauté de coélaboration de connaissances dans un mode de formation hybride afin de favoriser la cocréation de connaissances? Les recommandations qui découlent de cette analyse concernent les différents processus de régulation, les fonctionnalités des outils numériques et les influences mutuelles des contextes de coélaboration. Enfin, deux outils numériques permettant à la communauté d'assurer une régulation partagée seront présentés.

Mots-clés : communauté, coélaboration de connaissances, dispositif hybride, régulation 
As part of a graduate course, six students in Educational Studies engaged in a knowledgebuilding process, in-class and online, using the Knowledge Forum and Google Documents, around the theme of digital equity. Following this experience, one question arose: how can we maximize a hybrid knowledge building community's functioning to foster knowledge co-creation? This reflection includes the different regulation processes, the affordances of digital tools, and the mutual influences of knowledge building contexts. Two digital tools to facilitate regulation within the community are presented.

Keywords: community, knowledge building, hybrid learning environment, regulation

\section{RESUMEN}

Como parte de un curso de postgrado universitario, seis estudiantes de educación desarrollaron conjuntamente el tema de la equidad digital en el entorno en línea y presencial utilizando el Foro de Conocimiento y Google Drive. Tras esta experiencia, surgió la pregunta siguiente: ¿cómo podemos maximizar el funcionamiento de una comunidad híbrida de codesarrollo de conocimiento para favorecer la cocreación de dicho conocimiento? Las recomendaciones resultantes de este análisis se refieren a los diferentes procesos de regulación, a las ventajas de las herramientas digitales y a las influencias mutuas de los contextos de codesarrollo. Por último, se propondrán dos herramientas digitales para facilitar una regulación compartida en la comunidad.

Palabras clave: comunidad, codesarrollo de conocimiento, dispositivo híbrido, regulación

\section{Introduction}

Un des avantages majeurs offerts par le contexte numérique est celui de favoriser la collaboration. Les environnements numériques d'apprentissage facilitent la collaboration entre les différents acteurs impliqués dans l'apprentissage. Le numérique permet d'avoir accès à différents dispositifs pour collaborer partout et en tout temps : communication orale et écrite en mode synchrone et asynchrone, rédaction simultanée par plusieurs auteurs, suivi des modifications, etc. Or, les différents dispositifs ne garantissent pas pour autant un engagement des apprenants dans la collaboration et plusieurs facteurs influenceront la qualité de la collaboration. Aux facteurs technologiques des environnements numériques pouvant influencer la collaboration s'ajoutent les facteurs humains. L'avancement des connaissances peut tirer profit de la collaboration, chaque personne devenant une source potentielle de compréhension et d'information pouvant être partagée. Dans ce contexte, la collaboration se présente comme un important vecteur de l'apprentissage, comme c'est le cas pour la coélaboration de connaissances. La coélaboration de connaissances signifie que les apprenants collaborent à la construction des connaissances dans un objectif de progression à la fois individuel et collectif (Scardamalia, 2002). La communauté devient responsable de l'apprentissage et de la création de connaissances. Dans cet article, nous proposons de répondre à la question suivante : comment maximiser le fonctionnement d'une communauté de coélaboration de connaissances dans le cadre d'une formation hybride afin de favoriser la cocréation de connaissances? Ce texte décrit une expérience concrète de coélaboration de connaissances dans un contexte hybride d'apprentissage, combinant des interactions en présence et à distance. 


\section{Contexte}

À l'automne 2015, six étudiants de deuxième et troisième cycles en sciences de l'éducation, dont les deux auteures de cet article, ont été invités à prendre part à une communauté de coélaboration de connaissances portant sur l'équité numérique. Ils ont participé à un processus expérientiel dans lequel la négociation de sens, à des fins de compréhension collective, était effectuée dans le respect des interprétations individuelles de diverses sources d'autorité (Laferrière, 2015). Cette négociation de sens, axée sur des problèmes authentiques et réels, est au cœur du processus de coélaboration de connaissances (Bereiter et Scardamalia, 2003). Ainsi, la coélaboration de connaissances s'inscrivait à la fois comme objet d'étude et comme approche pédagogique.

Le séminaire était offert en mode hybride (en présence et en ligne), exigeant une présence physique minimale à trois moments spécifiques : au début, au milieu et à la fin de la session. Le séminaire combinait des interactions verbales et écrites au moyen d'outils numériques (Hutchison et Colwell, 2012). La participation à distance en mode synchrone était permise par l'intermédiaire de la plateforme Via de SVI eSolutions. Les participants ont privilégié les rencontres en présence chaque semaine, à l'exception d'une semaine où le travail à distance en mode synchrone a été expérimenté. Tout au long du séminaire, la participation en présence a été bonifiée par une participation à distance prenant la forme de contributions écrites en mode asynchrone grâce à différents outils numériques. Ces contributions ont joué un rôle majeur dans la collaboration autant en présence qu'à distance tout au long de la session.

\section{Le contexte pédagogique : la communauté de coélaboration de connaissances}

S'inspirant du modèle de la communauté d'apprentissage, une communauté de coélaboration de connaissances se préoccupe de problèmes qui surviennent réellement et qui touchent ses membres de près (Scardamalia et Bereiter, 1999). Si l'apprentissage est un processus par lequel le capital culturel de la société est distribué, la coélaboration de connaissances concerne quant à elle l'effort pour augmenter ce capital culturel (Scardamalia et Bereiter, 2003). Ainsi, le rôle de l'élève passe de celui d'un consommateur à celui d'un créateur (Martel, 2005).

Les membres se penchent sur une question ou un problème qu'ils examinent sous différentes perspectives et différents points de vue (Laferrière, 2005). Douze principes guident une communauté de coélaboration de connaissances ${ }^{1}$ (Scardamalia, 2002) :

1. Un savoir communautaire, une responsabilité collective

2. Utilisation constructive de sources d'autorité

3. Évaluation simultanée, ancrée et transformative

4. Démocratisation du savoir

5. Démarche épistémologique

6. Diversité des idées

7. Idées perfectibles

8. Ubiquité de l'élaboration de connaissances

9. Idées réelles, problèmes authentiques

10. Intégration des idées débattues et émergence de nouvelles idées

11. Discours transformatif

12. Avancement symétrique du savoir

${ }^{1}$ Traduction de S. Allaire tirée de la page Principes de coélaboration de connaissances : http://www.telelearning-pds.org/u/pv/princocons.htm 
Chaque principe présente à la fois une dimension sociocognitive et une dimension technologique. La dimension sociocognitive réfère à la dynamique sous-jacente à l'espace de connaissance qui est coconstruite par les participants (Zhang et al., 2007), tandis que la dimension technologique réfère aux exigences fonctionnelles de l'environnement numérique.

La communauté s'est engagée dans un processus de coélaboration de connaissances d'une durée de quinze semaines autour du thème de l'équité numérique, soutenue par des outils technologiques.

\section{Le contexte technologique : Knowledge Forum et Google Documents}

Pour soutenir la communauté de coélaboration de connaissances, autant lors des échanges en ligne qu'en présence, deux outils numériques ont été utilisés : Knowledge Forum et Google Documents. Le premier a été intégré à la démarche du cours, alors que le deuxième a été utilisé à l'initiative des étudiants. Nous en présentons ici les principales fonctionnalités ayant été mises à profit.

\section{KNOWLEDGE FORUM}

Knowledge Forum est un outil de création de forums ayant comme particularité qu'il possède une structure neuronale (un ensemble de notes liées les unes aux autres) plutôt qu'une structure linéaire. Les contributions, appelées « notes », peuvent ainsi être élaborées à partir d'une ou de plusieurs autres notes, aboutissant à un réseau de notes (figure 1).

\section{Figure 1}

Exemple de la structure neuronale des notes sur Knowledge Forum

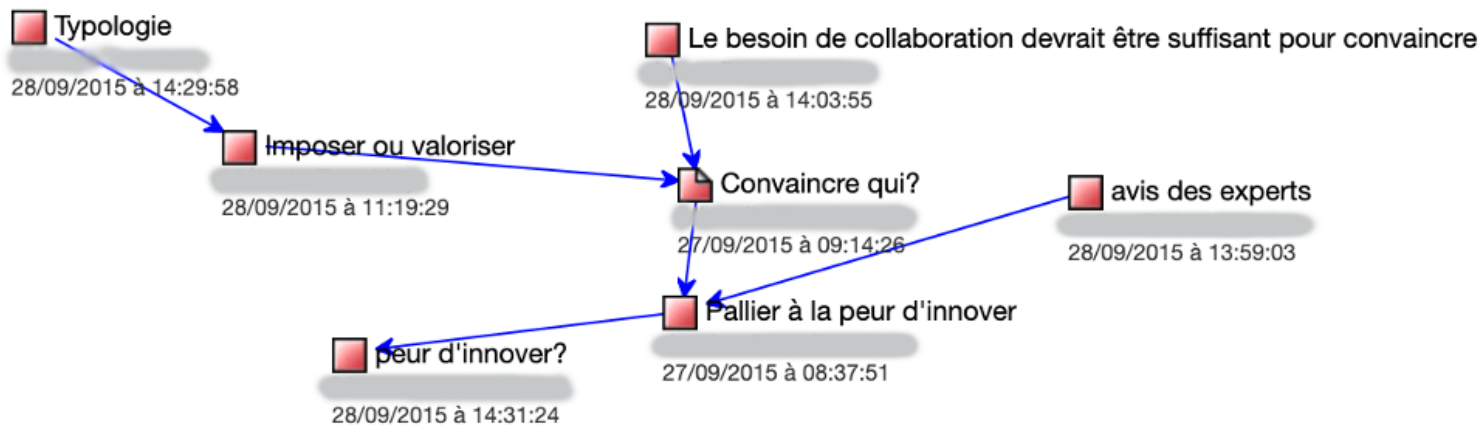

Au besoin, des notes de type "Élever le propos" (Rise above) peuvent être utilisées. Ces notes sont le résultat d'un travail collectif où sont synthétisées les idées développées et ayant mené à un certain consensus.

La fonctionnalité d'échafaudage a également soutenu le processus de coélaboration. Un échafaudage est le soutien qui permet aux apprenants de réaliser une tâche qu'ils ne pourraient pas accomplir sans aide (Belland, 2014). Ce soutien peut être fourni par un enseignant, un pair ou un outil numérique. Les échafaudages proposent des mécanismes de questionnement, de modélisation de processus, de rétroaction, etc.

Dans le cadre du séminaire, le répertoire d'échafaudage utilisé était de l'ordre de l'amélioration des idées. Les huit échafaudages permettant l'amélioration d'idées, choisis à l'avance par la responsable du cours parmi le répertoire d'échafaudages, pouvaient être utilisés par les membres de la communauté pour baliser 
certaines portions d'une note au moment de sa rédaction. Parmi les échafaudages, quatre relevaient davantage d'une démarche individuelle («Mon idée », " J'ai besoin de comprendre », " Je prends du recul », "Ce qu'en disent les experts ») et quatre autres, d'une démarche collective de la communauté ( " J'enrichis l'idée », " J'explore une idée différente », « Mettons notre savoir en commun », " Ce que nous savons maintenant »). La figure 2 présente un exemple de contribution rédigée à partir des échafaudages " Mon idée », " Ce qu'en disent les experts » et « Je prends du recul ».

\section{Figure 2}

Exemple de contribution utilisant les échaudages pour l'amélioration d'idées

\section{Valoriser comment?}

Créé(e) par: Michelle Deschenes

Lire Éditer Auteur(s) Liens Historique Pièce(s) jointe(s) Propriétés
Mon idée - II existe un mouvement en ce moment sur les badges en éducation (voir l'article de la Vitrine
Technologie Éducation). Je me questionne quant à la possibilité d'utiliser les badges pour valoriser le développement
professionnel dans des contextes non formels ou informels, en particulier dans des communautés de pratique. -

Ce qu'en disent les experts - Dans cet autre article, on définit le badge de la façon suivante : « Un badge est un dispositif numérique de reconnaissance de connaissances, de compétences ou de réalisations. Il est composé d'une image et d'une série de métadonnées servant à identifier l'émetteur du badge, son récipiendaire et les conditions d'obtention. Les badges sont conçus de façon à constituer une forme de portfolio dont le sac à badges de Mozilla, un pionnier et un chef de file en la matière, permet la collecte. Ils peuvent aussi être partagés via les réseaux sociaux comme Linkedln, Facebook ou Twitter. " -

Je prends du recul - J'ai récemment reçu un badge pour un cours complété sur Coursera (une plateforme de $\mathrm{MOOC}$ ). Après avoir travaillé très fort pendant plusieurs heures, sur une période de plusieurs semaines, obtenir une petite image m'a paru plutôt décevant : j'avais l'impression qu'on me mettait une étoile dans mon cahier Canada... J'étais déçue de ne pas recevoir un "diplôme" ou quelque chose de plus officiel. Toutefois, dans un contexte où il n'y a pas déjà de certification, peut-être que ça pourrait être plus intéressant? Évidemment, pour que les badges prennent leur sens, il faut les "exposer". Je me demande s'il y a beaucoup d'enseignants qui seraient prêts à investir du temps dans la création d'un portfolio? -

\section{GOOGLE DOCUMENTS}

Les étudiantes et étudiants du séminaire ont été invités à participer à un évènement organisé par l'Institute for Knowledge Innovation and Technology (IKIT). Pour synthétiser les contributions, une note rédigée collectivement a été créée pour chacune des idées prometteuses, c'est-à-dire que les six étudiantes et étudiants ont négocié le contenu qui y était écrit. Malheureusement, Knowledge Forum ne permet pas la rédaction simultanée comme d'autres outils. Les participants se sont donc tournés vers Google Documents, qui permet à plusieurs personnes de rédiger un texte simultanément.

Outre la rédaction simultanée par plusieurs utilisateurs et les fonctionnalités relevant des outils de traitement de texte, les fonctionnalités recherchées par la communauté à ce stade étaient le suivi des modifications. Le suivi des modifications permet de consulter rapidement le contenu ajouté, supprimé ou modifié. De plus, il est possible d'ajouter des commentaires en identifiant des utilisateurs, afin d'attirer leur attention sur une portion de texte, par exemple. Enfin, Google Documents permet de consulter l'historique des modifications du document et, au besoin, de rétablir une version antérieure du document. À l'époque, ces fonctionnalités n'étaient offertes que dans Google Documents; or aujourd'hui, elles sont offertes dans d'autres outils comme Microsoft Word dans Office 365. 


\section{La démarche de coélaboration de connaissances}

La démarche de coélaboration de connaissances s'est déployée en trois phases progressives ayant chacune un objectif spécifique : 1) définir les intentions d'apprentissage individuelles et collectives, 2) dégager une compréhension collective de l'équité numérique et 3 ) déterminer les facteurs d'engagement pour le développement professionnel visant l'équité numérique, la dernière phase étant la poursuite d'une réflexion amorcée lors de la deuxième phase.

\section{PHASE 1 : INTENTIONS}

Cette phase s'est déroulée en début de session. Pour se familiariser avec les principes de coélaboration de connaissances, les participants ont été invités à faire des lectures préalables et à partager leurs intentions d'apprentissage. Ils ont rédigé individuellement une note sur Knowledge Forum dans laquelle ils se sont brièvement présentés et ont rédigé leurs objectifs.

Une note de type « Élever le propos » a spontanément été rédigée par une participante afin de synthétiser les objectifs individuels pour en dégager les thèmes d'intérêts collectifs: l'apprentissage en réseau, les environnements collaboratifs numériques et la régulation. Les participants ont convenu que, pour favoriser la collaboration, il faut avoir la perception que seul, il sera impossible d'y arriver. C'est ce qui s'est produit lorsque les participants ont mis à profit leur contexte respectif au service de l'avancement de la réflexion collective.

\section{PHASE 2 : ÉQUITÉ NUMÉRIQUE}

La deuxième phase correspond au travail de réflexion effectué en présence (durant la majorité des séances) et à distance (de manière synchrone lors d'une séance et asynchrone entre chacune des séances) autour du thème de l'équité numérique. Vingt-trois notes ont d'abord été rédigées puis utilisées pour "Élever le propos" visant à définir trois niveaux d'équité du point de vue 1 ) des infrastructures et ressources numériques, 2 ) des enseignants et 3 ) des apprenants. Ces niveaux ont été définis en précisant les acteurs impliqués, les enjeux, les recommandations et les actions à poser. Le livrable de cette première démarche a été utilisé pour nourrir la discussion sur l'équité numérique à l'EDUsummlt 2015, en Thaïlande.

Cette expérience de coélaboration de connaissances a permis d'avoir un aperçu du potentiel de cette démarche. Elle a permis par ailleurs d'identifier que l'accès aux ressources n'est pas suffisant pour garantir l'équité numérique : il faut également des enseignants compétents dans l'utilisation pédagogique des outils numériques. Ce constat a mené les participants à l'importance du développement professionnel pour atteindre l'équité numérique.

\section{PHASE 3 : DÉVELOPPEMENT PROFESSIONNEL}

Le développement professionnel apparaissant comme un enjeu pour les deux derniers niveaux d'équité numérique (enseignants et apprenants), la démarche de la communauté s'est orientée autour de ce thème. Les participants ont effectué une analyse individuelle de communautés de développement professionnel existantes. Ils ont ensuite synthétisé les analyses dans une note collective en ciblant les idées prometteuses.

Le livrable de cette dernière phase était la participation à distance au Knowledge Building International au Brésil. Ce livrable a pris la forme « d'un tableau présentant les principes à considérer dans la structuration des communautés de pratique en ligne visant le développement professionnel dans une perspective d'équité numérique » (Tremblay et Dion-Routhier, 2018, p. 571). 


\section{Retour sur la démarche : maximiser le fonctionnement de la communauté pour favoriser la coélaboration de connaissances}

Ce récit de pratique s'inspire de l'approche ethnographique: bien que cette approche ne permette pas la généralisation, elle permet de décrire en profondeur le fonctionnement d'un groupe grâce à une observation fine et complexe d'une situation (Côté et al., 2020).

À différents moments, des difficultés se sont manifestées dans la communauté, nuisant au processus de coélaboration de connaissances. Plus la communauté avançait dans la démarche, plus ces difficultés étaient observées et nommées par les membres de la communauté lors des échanges synchrones. La première difficulté s'apparente aux différentes formes d'engagement pouvant cohabiter au sein de la communauté. Les membres de la communauté s'accordaient sur l'importance de l'engagement de chacun dans l'atteinte des objectifs établis. Pour pallier cette difficulté et atteindre l'objectif collectif, différents processus de régulation, présentés dans la section suivante, ont dû être mis en place. La deuxième difficulté nommée par les membres de la communauté lors des échanges et des processus de régulation concerne les avantages et inconvénients des différents modes d'interaction induits par le contexte de formation hybride. Afin de tirer le meilleur des outils et des modes d'interaction en présence et à distance, les participants se sont inspirés de certains avantages d'un contexte afin de les transposer à l'autre contexte.

\section{Mettre à profit les différents processus de régulation}

Järvelä et Hadwin (2013) indiquent que, dans une communauté, les processus de régulation dans un contexte de collaboration sont de trois types : l'autorégulation, la corégulation et la régulation partagée. L'autorégulation $(m y)$ est celle où chaque membre du groupe prend la responsabilité de réguler ses propres apprentissages en adoptant, en développant et en affinant ses stratégies. L'autoévaluation est une composante importante de l'autorégulation (Zimmerman, 1990). Elle permet de comparer l'état actuel avec un état désiré, autrement dit de comparer la performance avec les objectifs fixés; les objectifs sont d'ailleurs jugés fondamentaux dans le processus de régulation (Järvelä et Hadwin, 2013). L'autorégulation prévoit qu'on « anticipe et élabore des stratégies, les évalue et les ajuste en fonction des résultats obtenus afin de mener à bien ses apprentissages » (Jézégou, 2015, p. 6). Zimmerman (2008) distingue deux types d'autorégulation : proactive (créer des buts et des plans d'action) et réactive (surmonter des obstacles).

En corégulation (your), chaque membre aide les autres membres du groupe à réguler leurs apprentissages, soucieux de leurs objectifs et de leur progression, en plus de soutenir le développement des habiletés et des stratégies d'autorégulation des autres. La régulation partagée (our) est présente lorsque le groupe régule collectivement les buts et les tâches partagés ainsi que les processus d'apprentissage (Järvelä et Hadwin, 2013). Une régulation réussie se manifeste par une adaptation stratégique en réponse à un défi ou une situation problématique (Winne et Hadwin, 2008). Les sections qui suivent présentent les pivots stratégiques qu'a effectués la communauté lorsque cela s'est avéré nécessaire.

\section{L'AUTORÉGULATION}

Bien que présente à différents niveaux tout au long de la démarche, l'autorégulation s'est surtout manifestée à mi-parcours et à la fin, à l'aide d'autoévaluations. Deux autoévaluations documentées ont été demandées par la responsable du cours à des fins d'évaluations dans le cadre du séminaire. À partir des objectifs individuels et collectifs d'apprentissage, les étudiantes et étudiants devaient rédiger un texte dans lequel ils examinaient l'objet retenu et démontraient une capacité d'amélioration des idées reconnues 
prometteuses dans un texte. Les étudiants devaient référer aux niveaux de Stone Wiske (1998) pour s'autoévaluer: naïf (performances basées sur des connaissances intuitives), novice (performances enracinées dans les rituels de l'évaluation et de la scolarisation), apprenti (performances basées sur des connaissances et des modes de pensée disciplinaires), et maitre (performances intégrées, créatives et critiques).

\section{LA CORÉGULATION}

Le partage spontané (non exigé par la responsable) sur Knowledge Forum des intentions et objectifs de chacun des membres dès la première semaine a permis de faciliter la corégulation tout au long de la session. II était plus facile de mettre à la disposition des autres participants des ressources pouvant leur permettre d'atteindre leur objectif, puisqu'ils étaient connus et adoptés par les autres membres.

Bien que le processus de corégulation n'ait pas été formellement demandé, les participants ont pris l'initiative de partager également leurs autoévaluations dans Knowledge Forum. À la rencontre suivante (en présence), le groupe a pu réagir aux autoévaluations de chacun des membres, explorant à la fois le contenu de l'autoévaluation et la façon d'en présenter les réflexions et les résultats. L'un des principes de coélaboration de connaissances est l'avancement symétrique du savoir; nous pourrions y ajouter l'avancement symétrique des processus. Par exemple, les échanges au sujet des autoévaluations ont influencé les autoévaluations finales, à la fois en matière de démarches et de résultats.

\section{LA RÉGULATION PARTAGÉE}

À la lecture des différentes autoévaluations, quelques disparités ont été notées. Par exemple, qu'en était-il de l'autoévaluation de la communauté par la communauté? Les discussions se sont alors déplacées de l'individuel vers le collectif, effectuant alors une régulation partagée de la communauté. Les participants ont adopté un code de couleurs (rouge, jaune, vert) pour évaluer les performances de la communauté quant aux différents principes de coélaboration à l'aide du prototype d'un outil développé par un membre de la communauté et que nous présenterons dans les prochaines sections. La régulation partagée de la communauté a permis aux membres de mieux s'approprier les principes de coélaboration, autant leur dimension sociocognitive que leur dimension technologique. Cette étape a contribué à raffiner la dernière autoévaluation réalisée par chacun des participants au terme de la session. Par ailleurs, le fait de négocier cette régulation en groupe a permis de cibler les principes qui étaient moins bien intégrés par la communauté, de même que les pistes d'amélioration possibles.

Par l'autorégulation, la corégulation et la régulation partagée, la communauté s'est inspirée des composantes de la régulation : la négociation d'objectifs et de normes guidant le travail, l'adoption et l'adaptation d'outils et de stratégies, le suivi du progrès, les interventions nécessaires pour éviter de s'éloigner du plan, et la persistance et l'adaptation face aux défis (Järvelä et Hadwin, 2013).

\section{Mettre à profit les influences mutuelles des contextes en présence et à distance}

Une des particularités de la communauté était les avantages offerts par l'environnement numérique et le mode à distance. La communauté s'est adaptée en transposant les avantages d'un contexte à l'autre, palliant ainsi les limites perçues en présence et à distance. Par exemple, lors des séances en présentiel, des consensus étaient dégagés, concernant autant le contenu que la démarche à poursuivre avant la prochaine séance. Or le délai (quelques jours) avant de faire le travail prévu permettait d'approfondir la réflexion, creusant parfois un écart entre la compréhension individuelle de chacun. Par conséquent, il a été proposé que la communauté se dote d'une démarche s'inspirant d'un avantage du contexte à distance, soit la fonctionnalité « Élever le propos » du Knowledge Forum. Au terme de chaque séance en présentiel, 
un membre, différent chaque fois, a rédigé une note qui présentait à la fois une synthèse des idées ayant émergé durant la séance et le résultat de la négociation de ce qui devait être produit par la suite.

Parallèlement, les interactions à distance ont permis de soutenir les échanges en présence. À plusieurs reprises, les participants ont transféré dans leurs interactions en présentiel les pratiques de coélaboration développées en ligne, notamment avec les échafaudages et les fonctionnalités du Knowledge Forum ("Élever le propos », "Ce qu'en disent les experts », " Je prends du recul », etc.). Ce réinvestissement dans le discours oral a permis de soutenir les échanges et le processus de coélaboration de connaissances.

\section{Soutenir l'autorégulation et la régulation partagée avec le numérique}

Bien que le contexte dans lequel la communauté évoluait était hybride, les processus de régulation ont été réalisés dans une forme relativement traditionnelle : des autoévaluations individuelles et des échanges en présentiel. Les membres de la communauté ont cependant mis le numérique à profit en utilisant le prototype d'un outil numérique qui a évolué depuis. Poursuivant notre réflexion et reconnaissant l'intérêt des outils numériques pour favoriser les interactions de la communauté hybride, nous nous sommes intéressées aux fonctionnalités pouvant soutenir les différents processus de régulation. Les deux outils suivants sont issus de cette démarche.

Le premier outil ${ }^{2}$ a été créé sur mesure par une des auteures (MD) et le prototype a été utilisé par la communauté lors du séminaire. Avec cet outil, les membres d'une communauté peuvent évaluer leur niveau de maitrise des différents principes d'une communauté de coélaboration de connaissances, dans une démarche individuelle ou collective. Les principes sont successivement présentés et définis (figure 3). Pour chacun d'eux, l'atteinte du niveau de maitrise est négociée parmi les membres puis sélectionnée de manière interactive à l'aide des niveaux de Stone Wiske (1998), une seule personne pouvant effectuer le choix.

\section{Figure 3}

Autoévaluation et évaluation partagée avec un outil créé sur mesure

\footnotetext{
${ }^{2}$ L'outil est accessible gratuitement à l'adresse http://mdeschenes.com/regulation.
} 
Les résultats sont ensuite présentés dans un graphique polaire (figure 4), où les évaluations entrées sont affichées pour chacun des principes de la coélaboration. Les couleurs sont utilisées pour afficher le regroupement des principes selon les cinq principes simplifiés (Allaire et Lusignan, 2011) :

1. En bleu : collaboration et complémentarité des idées à partir de questions réelles et de problèmes authentiques (principe 9).

2. En mauve: amélioration et diversification des idées de manière participative par le discours (principes 6, 7 et 11).

3. En rouge : responsabilisation des élèves dans un esprit démocratique (principes 1, 4, 5, 8, et 12).

4. En jaune: considération de sources fiables tout au long de la démarche d'investigation (principe 2).

5. En vert : évaluation partagée, en contexte, tout au long du processus (principes 3 et 10).

\section{Figure 4}

Résultat d'une autoévaluation ou d'une évaluation partagée de la communauté

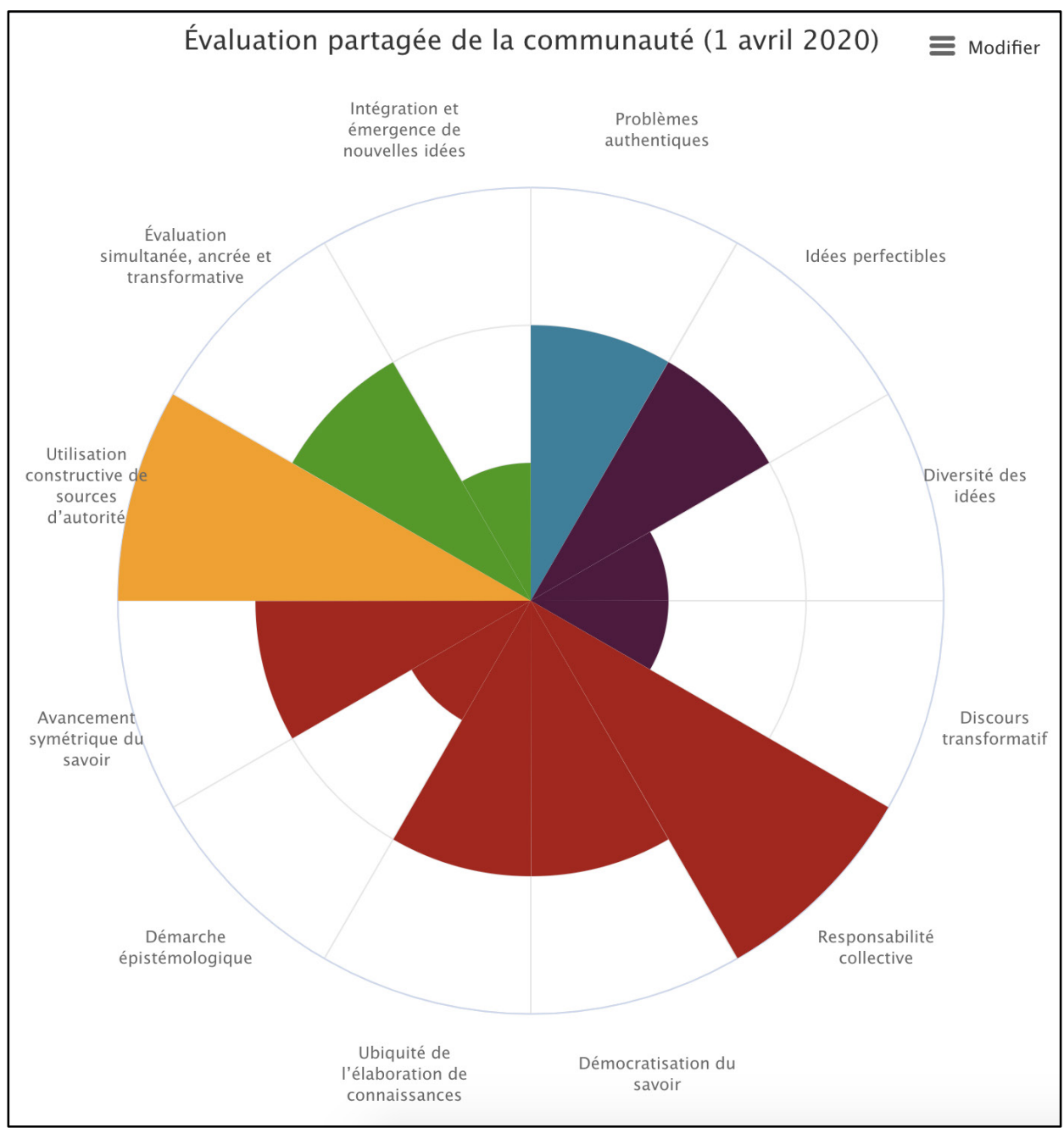


L'outil a été utilisé dans une petite communauté; le nombre de membres étant limité, la négociation du niveau d'atteinte était possible. Or, la négociation d'une telle évaluation partagée dans une communauté regroupant un plus grand nombre de membres peut s'avérer plus complexe. C'est pourquoi un second outil $^{3}$ a été développé par une des auteures (MT) pour l'utilisation en grande communauté (30 à 40 membres). Avec cet outil, les membres sont appelés à entrer individuellement leur évaluation de la communauté selon les douze principes de coélaboration (figure 5), puis la répartition des réponses est affichée (figure 6), mettant en œuvre le processus de négociation lorsqu'il est nécessaire.

\section{Figure 5}

Principe de coélaboration présenté à la communauté pour des fins d'évaluations

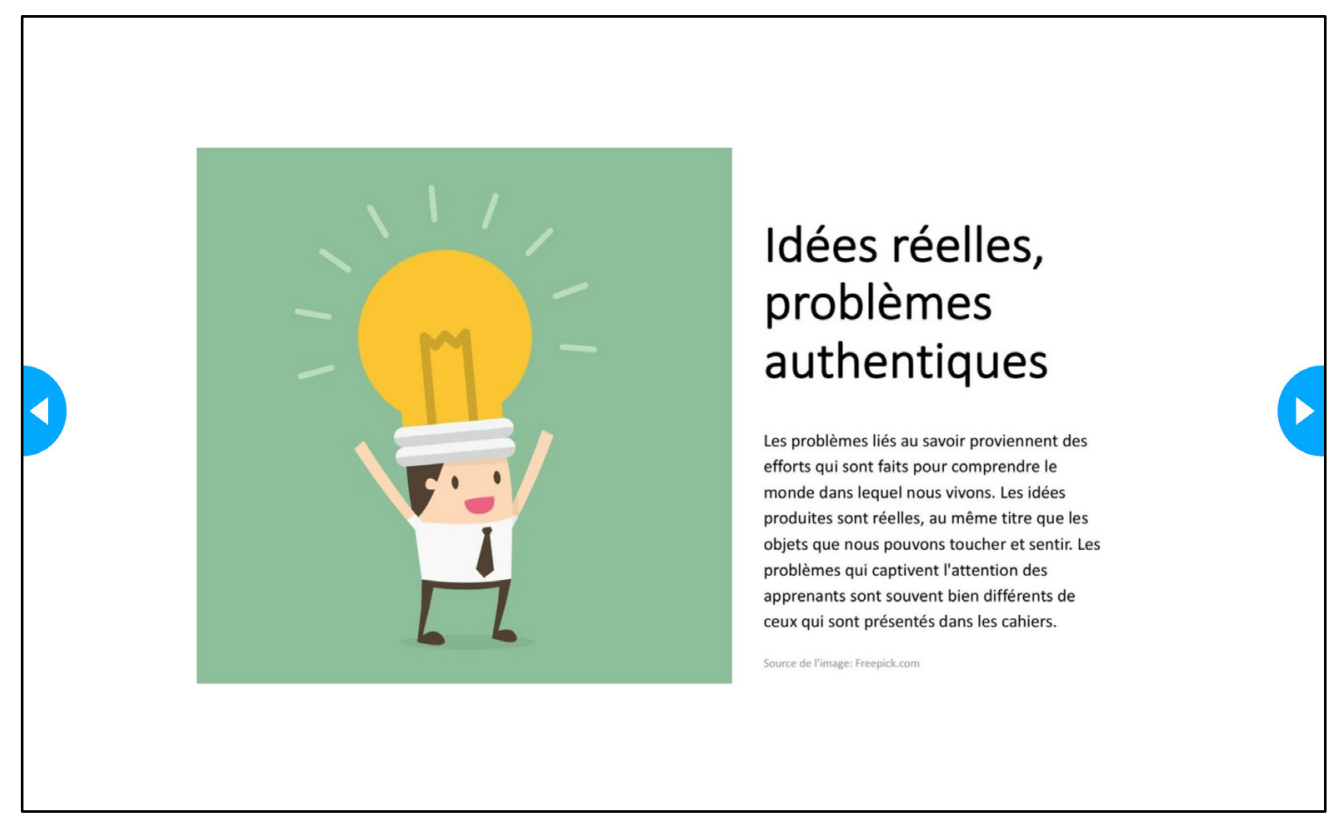

${ }^{3}$ L'outil est disponible à l'adresse https://share.nearpod.com/e/MvPmNAYyP4. La création d'un compte Nearpod est nécessaire. 


\section{Figure 6}

Résultat d'une évaluation partagée de la communauté

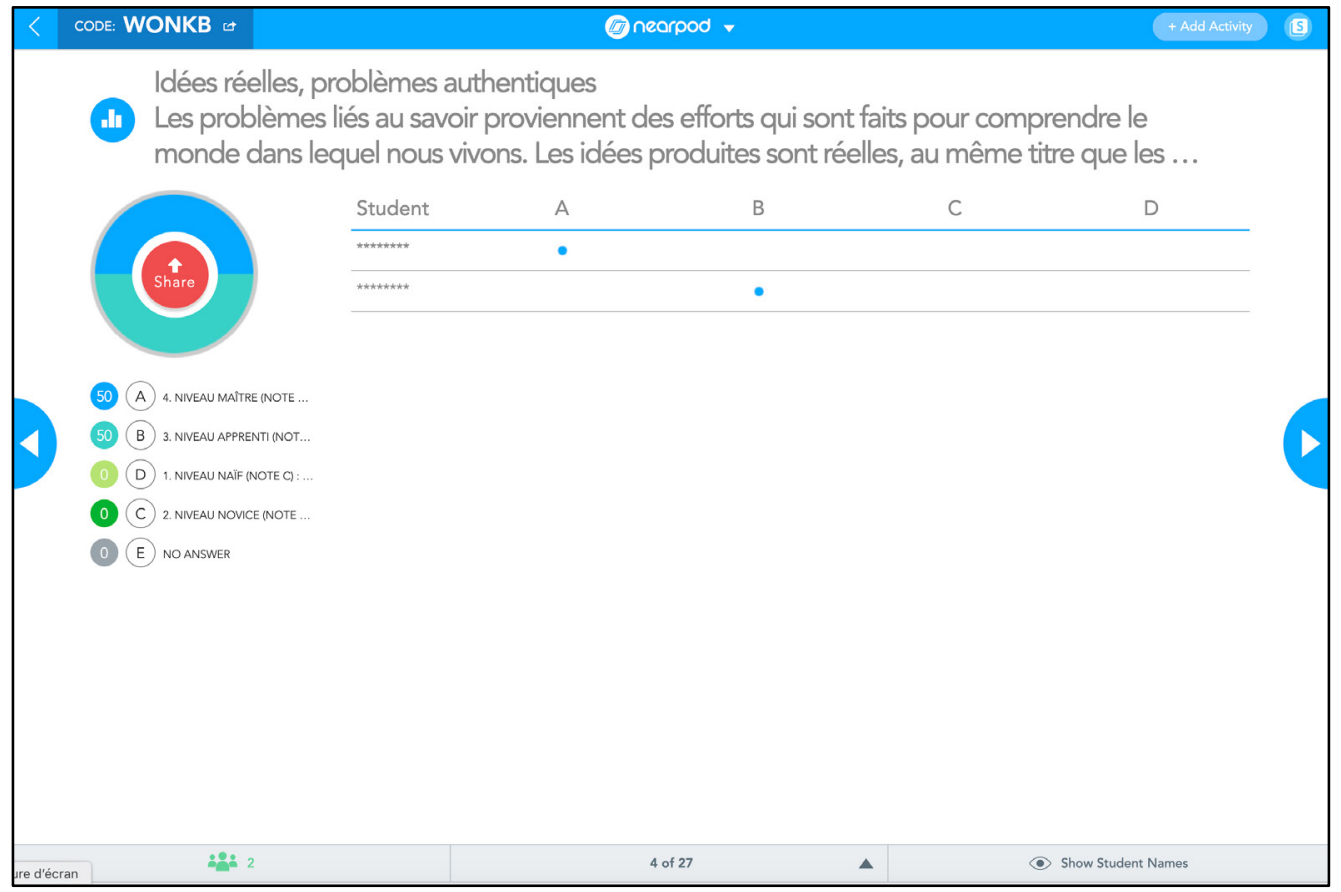

Au-delà de l'acte d'évaluation individuelle, c'est la négociation de la compréhension des principes et leur transposition dans le contexte de la communauté qui est intéressante dans une perspective de régulation. Les outils proposés permettent ainsi de prendre " une capture» de la maitrise des principes par la communauté. Ces outils gagnent à être utilisés à plusieurs moments afin d'apprécier la variation et la progression.

\section{Conclusion}

L'expérience de coélaboration de connaissances vécue par la communauté a permis aux membres de saisir l'importance de l'influence du contexte dans une situation d'apprentissage. Bien que convaincues de l'immense potentiel de l'approche, nous avons été en mesure de constater les facteurs humains et technologiques pouvant avoir une incidence sur l'expérience de cette approche et sur les résultats de la démarche. En cohérence avec l'approche de communauté de coélaboration de connaissances, la communauté s'est engagée à formaliser les modes de régulation à l'aide des principes de cette approche et à enrichir les interactions en s'inspirant des avantages offerts par différents outils numériques. Les résultats de cette réflexion nous ont menées à la création d'outils de régulation visant à favoriser la collaboration.

Il demeure que la négociation des résultats de ce type de régulation partagée gagnerait à se faire en tirant profit des fonctionnalités d'analyses présentes dans les outils numériques utilisés pour soutenir une communauté de coélaboration de connaissances. Dans l'environnement Knowledge Forum par exemple, il serait pertinent d'analyser l'évolution de l'utilisation des échafaudages au fil de la démarche. Cette prise de mesure pourrait être l'occasion pour la communauté de rectifier la démarche si les échafaudages manifestant un processus individuel étaient privilégiés aux dépens de ceux manifestant un processus 
collectif. De plus, une telle analyse ne devrait pas se limiter à une évaluation quantitative des interventions, mais plutôt à un examen du caractère contributif de celles-ci. Par ailleurs, notons que l'utilisation d'un outil comme ceux proposés ne devrait remplacer ni la négociation entre les participants ni la créativité dont ils pourraient faire preuve. II s'agit pour la communauté de déterminer l'équilibre souhaité et tirer profit de la tension créée entre la structure et la créativité (Brennan, 2012).

\section{Liste de références}

Allaire, S. et Lusignan, G. (2011). Enseigner et apprendre en réseau : collaborer entre écoles distantes à l'aide des TIC. Anjou : Éditions CEC.

Belland, B. R. (2014). Scaffolding: Definition, Current Debates, and Future Directions. Dans J. M. Spector, M. D. Merill, J. Elen et M. J. Bishop (dir.), Handbook of Research on Educational Communications and Technology $\left(4^{\mathrm{e}}\right.$ éd., p. 505-518). New York : Springer.

Bereiter, C. et Scardamalia, M. (2003). Learning to work creatively with knowledge. Dans E. De Corte, L. Verschaffel, $\mathrm{N}$. Entwistle et J. van Merriënboer (dir.), Unravelling basic components and dimensions of powerful learning environments. EARLI Advances in Learning and Instruction Series (p. 55-68). Oxford, UK: Elsevier Science.

Brennan, K. (2012). Best of both worlds: Issues of structure and agency in computational creation, in and out of school (thèse de doctorat). Massachusetts Institute of Technology, Cambridge, MA.

Côté, D., Dubé, J. et Arsenault, M. (2020). L'approche ethnographique : illustration dans un contexte de réadaptation au travail. Dans M. Corbière et N. Larivière (dir.), Méthodes qualitatives, quantitatives et mixtes ( $2^{\mathrm{e}}$ éd., p. 59-88). Québec : Presses de l'Université du Québec.

Hutchison, A. et J. Colwell, J. (2012). Using a wiki to facilitate an online professional learning community for induction and mentoring teachers. Education and Information Technologies 17(3), 273-289. https://doi.org/10.1007/s10639-011-9159-7

Järvelä, S. et Hadwin, A. F. (2013). New Frontiers: Regulating Learning in CSCL. Educational Psychologist, 48(1), 25-39.

Jézégou, A. (2015). Diriger soi-même ses activités d'apprentissage par et dans un Mooc de type connectiviste. International Journal of E-Learning \& Distance Education, 30(1). http://www.ijede.ca/index.php/jde/article/view/868/1574

Laferrière, T. (2005). Les communautés d'apprenants en réseau au bénéfice de l'éducation, Encounters on Education, 6, 5-21. https://qspace.library.queensu.ca/bitstream/1974/480/1/art\%201\%20laferriere.pdf

Laferrière, T. (2015). TEN-7000 : Apprentissage en réseau. Plan de cours inédit, Université Laval.

Martel, V. (2005). Émergence d'une communauté d'apprentissage en réseau à l'ordre primaire: l'activité de transformation d'un environnement d'apprentissage par la direction, les enseignants et les élèves (étude de cas) [thèse de doctorat inédite]. Université Laval, Québec, Canada.

Scardamalia, M. (2002). Collective cognitive responsability for the advancement of knowledge. Dans B. Smith (Ed.), Liberal Education in a Knowledge Society (p. 67-98). Peru, ILOpen Court. http://ikit.org/fulltext/2002CollectiveCog.pdf.

Scardamalia, M. et Bereiter, C. (1999). Schools as knowledge building organizations. Dans D. Keating et C. Hertzman (dir.), Today's children, tomorrow's society: The developmental health and wealth of nations (p. 274-289). New York: Guilford.

Scardamalia, M., et Bereiter, C. (2003). Knowledge Building. Dans J. W. Guthrie (dir.), Encyclopedia of Education ( $2^{\mathrm{e}}$ éd., p. 1370-1373). New York : Macmillan Reference, USA.

Stone Wiske, M. (1998). Teaching for understanding: Linking research with practice. San Francisco: Jossey-Bass Publishers.

Tremblay, M. et Dion-Routhier, J. (2018). Coélaboration de connaissances sur les facteurs d'engagement à une communauté de pratique pour le DP (CoDP). McGill Journal of Education/Revue des sciences de l'éducation de McGill, 53(3).

Winne, P. et Hadwin, A. (2008). The weave of motivation and self-regulated learning. Dans D. Schunk et B. Zimmerman (dir.), Motivation and self-regulated learning (p. 297-314). New York : LawrenceErlbaum.

Zhang, J., Scardamalia, M., Lamon, M., Messina, R. et Reeve, R. (2007). Socio-cognitive dynamics of knowledge building in the work of 9- and 10-year-olds. Educational Technology Research and Development, 55(2), 117-145. https://doi.org/10.1007/s11423-006-9019-0 
Zimmerman, B. J. (1990). Self-regulated learning and academic achievement: An overview. Educational Psychologist, 25(1), 3-17.

Zimmerman, B. J. (2008). Goal Setting: A Key Proactive Source of Academic Self-Regulation*. Dans D. H. Schunk et B. J. Zimmerman (dir.), Motivation and self-regulated learning: Theory, Research, and applications (p. 267-295). New York, New York, USA: Lawrence Erlbaum Associates. 